\title{
ON THE SEMISIMPLICITY OF GROUP ALGEBRAS
}

\author{
ORLANDO E. VILLAMAYOR ${ }^{1}$
}

In this paper we find sufficient conditions for a group algebra over a commutative ring to be semisimple. In particular, the case in which the group is abelian is solved for fields of characteristic zero, and, in a more general case, for semisimple commutative rings which are uniquely divisible by every integer. Under similar restrictions on the ring of coefficients, it is proved the semisimplicity of group algebras when the group is not abelian but the factor group module its center is locally finite. ${ }^{2}$

In connection with this problem, we study homological properties of group algebras generalizing some results of M. Auslander [1, Theorems 6 and 9]. In fact, Lemmas 3 and 4 give new proofs of Auslander's Theorems 6 and 9 in the case $C=(1)$.

1. Notations. A group $G$ will be called a torsion group if every element of $G$ has finite order, it will be called locally finite if every finitely generated subgroup is finite and it will be called free (or free abelian) if it is a direct sum of infinite cyclic groups.

Direct sum and direct product are defined as in [3]. Given a set of rings $R_{i}$, their direct product will be denoted by $\Pi R_{i}$.

If $G$ is a group and $R$ is a ring, the group algebra generated by $G$ over $R$ will be denoted by $R(G)$.

In a ring $R$, radical and semisimplicity are meant in the sense of Jacobson [5].

A ring is called regular in the sense of von Neumann [7]. It is well known that every regular ring is semisimple.

All algebras and rings will be assumed to have a unit and field will mean commutative field. Modules over rings will always be unitary left-modules unless otherwise expressed.

In homological terminology and notations we will follow [3]. In order to avoid confusions when a ring $A$ may be considered as an algebra over different ground rings, we shall denote by $S$-dim. $A$ (resp. $S$-w. $\operatorname{dim} . A$ ) the dimension (resp. w. $\operatorname{dim}$ ) of the $S$-algebra $A$.

Received by the editors November 6, 1957 and, in revised form, December 31, 1957.

${ }^{1}$ This paper was supported by a J. S. Guggenheim fellowship.

${ }^{2}$ We would like to express our thanks and indebtedness to Professor Rosenberg for suggestions that have simplified the presentations of these results. 
2. Our results are consequences of the following lemmas.

Lemma 1. Let $R$ be a ring and $S$ a subring contained in the center. If $S$-w. $\operatorname{dim} R=0, R$ is $S$-projective and $S$ is semisimple, then $R$ is semisimple.

Proof. Since $S$ is commutative and semisimple, then it is subdirect sum of fields $F_{i}$ ( $i \in M$, an arbitrary set), that is, $S \subseteq \prod F_{i}$ and $\phi_{i}: S \rightarrow F_{i}$ are epimorphisms induced by the projections $\psi_{i}: \prod F_{j} \rightarrow F_{i}$.

We wish to show, in first place, that $R \subseteq \prod R \otimes_{S} F_{i}$. Since $R$ is $S$ projective the exactness of

$$
0 \rightarrow S \rightarrow F_{i} \Pi
$$

yields, by tensoring with $R$, the exactness of

$$
0 \rightarrow R \rightarrow R \otimes \otimes_{S} \prod F_{i} .
$$

Now there is a natural map from $R \otimes_{S} \prod F_{i}$ to $\Pi R \otimes_{S} F_{i}[3$, p. 32]. This map is a monomorphism when $R$ is $S$-projective; indeed, this is clear when $R$ is $S$, hence, since $\otimes$ commutes with direct sums, when $R$ is $S$-free, and so finally when $R$ is $S$-projective.

Now, since every sequence $S \rightarrow F_{i} \rightarrow 0$ is exact, then $R \rightarrow R \otimes_{S} F_{i} \rightarrow 0$ is exact, hence, $R$ is subdirect sum of the rings $R \otimes_{S} F_{i}$.

Since $R$ is $S$-projective, the spectral sequence $(5 \mathrm{a})$ of $[3$, p. 347] shows that w.gl.dim $R \otimes_{S} F_{i} \leqq S$-w. $\operatorname{dim} R+$ w. gl. $\operatorname{dim} F_{i}$. Now, $F_{i}$ is a field and $S$-w. $\operatorname{dim} R=0$ by hypothesis, thus w. gl. $\operatorname{dim} R \otimes_{S} F_{i}=0$. But, by [4, Theorem 5] this implies $R \otimes_{S} F_{i}$ is regular, hence semisimple. Then $R$ is a subdirect sum of semisimple rings and so semisimple.

Concerning homological dimension of group algebras we have:

Lemma 2. Let $G$ be a group and $C$ a subgroup contained in the center of $G$. If $K$ is any commutative ring, then $K(C)-\operatorname{dim} K(G)=0$ if and only if: (1) $G / C$ is finite, (2) $K$ is uniquely divisible by the order of $G / C$.

Proof. Since $K(G)$ is a free $K(C)$-module, then [6, Theorem 1, p. 88] $K(C)-\operatorname{dim} K(G)=0$ implies that the order of $K(G)$ as $K(C)$ module is finite. Since it equals the order of $G / C$, the necessity of the finiteness of $G / C$ is proved. The necessity of (2) is a consequence of Lemma 4 below.

Suppose, now, that conditions (1) and (2) are fulfilled. Let us call $G^{*}=K(G) \otimes_{K(C)} K(G)^{*}$.

In order to show $K(C)-\operatorname{dim} K(G)=0$, we apply [3, IX, 7, 7]. In first place, we see that the elements $a \otimes a^{-1}$ are independent of the choice of $a$ in the coset of $G$ modulo $C$. In fact, if $b=a c, c \in C$, then 
$b \otimes b^{-1}=a c \otimes c^{-1} a^{-1}$ and, since we tensor over $K(C), b \otimes b^{-1}=a c c^{-1}$ $\otimes a^{-1}=a \otimes a^{-1}$.

Let $m$ be the order of $G / C$ and $\left\{a_{i}\right\}$ a complete set of representatives in $G$ of $G / C$. Let us call $\theta=(1 / m) \sum_{1} a_{i} \otimes a_{i}^{-1}$. If $\sigma: K(G) \otimes K(G)$ $\rightarrow K(G)$ is the natural homomorphism, then $\sigma(\theta)=e$ (the identity of $K(G))$.

Let $\left(a_{0}^{\prime}\right)$ be the cyclic group generated by $a_{0}^{\prime}$ (=the image of $a_{0}$ in $G / C)$, let $\left\{b_{k}^{\prime}\right\}$ be a complete set of representatives in $G / C$ of the (right-) cosets modulo $\left(a_{0}^{\prime}\right)$ and $\left\{b_{k}\right\}$ a complete set of representative of $\left\{b_{k}{ }^{\prime}\right\}$ in $G$. Then, $\left\{a_{0}^{j} b_{k}\right\}$ is a complete set of representatives of $G / C$ in $G$, and

$$
\theta=(1 / m) \sum_{j, k} a_{a_{0}^{j}}^{j} b_{k} \otimes b_{k}^{-1} a_{0}^{-j} .
$$

Then, given $a_{0} \in G$, we may write $\theta=\sum \theta_{k}$, with $\theta_{k}=\sum_{j} a_{0}^{y} b_{k}$ $\otimes b_{\boldsymbol{k}}^{-1} a_{0}^{-j}$, and, obviously, $a_{0} \theta_{k}=\theta_{k} a_{0}$, hence $a_{0} \theta=\theta a_{0}$ for every $a_{0} \in G$, then $\alpha \theta=\theta \alpha$ for every $\alpha \in K(G)$.

Since $\theta$ satisfies both conditions of [3, IX Proposition 7.7, p. 179] $K(C)-\operatorname{dim} K(G)=0$.

We shall use in the following lemma the fact that, in particular, $K(G)$ is $G^{e}$-flat.

Lemma 3. Let $G$ be a group and $C$ a subgroup contained in the center of $G$. If $G / C$ is locally finite and $K$ is a commutative ring, then $K(C)$ w. $\operatorname{dim} K(G)=0$ if and only if $K$ is uniquely divisible by the order of each element in $G / C$.

We shall prove, now, the sufficiency of our conditions.

$K(C)$-w.dim $K(G)=0$ means, by definition, that $K(G)$ is $G^{e}$-flat.

Suppose $\sum_{i} \lambda_{i} \alpha_{i}=0\left(\lambda_{i} \in G^{e}, \alpha_{i} \in K(G)\right)$. Let $\left\{a_{k}\right\}$ be the (finite) set of elements of $G$ appearing in this expression, and $S$ the subgroup of $G$ generated by $\left\{a_{k}\right\}$ over $C$.

Then, $\lambda_{i} \in S^{e}, \alpha_{i} \in K(S)$ and, under our hypothesis, $S / C$ satisfies the conditions of Lemma 2, hence $K(S)$ is $S^{e}$-flat, therefore, there are elements $\beta_{i j} \in K(S), \mu_{i j} \in S^{e}$ satisfying the conditions of [3, VI, Example 6, p. 123]. The inclusion $S \subseteq G$ implies that, actually, $\beta_{i j} \in K(G)$ and $\mu_{i j} \in G^{e}$, then $K(G)$ is $G^{e}$-flat and the sufficiency of the conditions of the lemma is proved.

The necessity is a consequence of the following lemma.

Lemma 4 . Let $G$ be a group and $C$ a subgroup contained in the center of $G$. If $K$ is a commutative ring, then $K(C)$-w. $\operatorname{dim} K(G)=0$ implies $G / C$ is a torsion group and $K$ is uniquely divisible by the order of each element in $G / C$. 
Proof. Suppose $G / C$ is not a torsion group, then there is at least one element $a^{\prime} \in G / C$ of infinite order. If $a \in G$ is a representative of $a^{\prime}$, it may be seen by direct computation that $1 \otimes a^{*}-a \otimes 1^{*}$ is not a divisor of zero. If 1 is the unit of $K(G)$, then $\left(1 \otimes a^{*}-a \otimes 1^{*}\right) \cdot 1=0$, and no elements $\lambda_{i} \in G^{e}$ verify the conditions of [3, VI, Example 6, p. 123], hence, $K(G)$ is not $G^{e}$-flat, that is, $K(C)$-w. $\operatorname{dim} K(G) \neq 0$.

Thus, $G^{\prime}=G / C$ is a torsion group. Let $a^{\prime} \in G^{\prime}$ have order $n$ and let $Z^{\prime}$ be the cyclic subgroup of $G^{\prime}$ generated by $a^{\prime}$. Let $\left\{b_{j}^{\prime}\right\}$ be a complete set of representatives of the left-cosets of $G^{\prime}$ modulo $Z^{\prime}$, i.e., $G^{\prime}=\cup_{k} Z^{\prime} b_{k}^{\prime}, Z^{\prime} b_{j}^{\prime} \cap Z^{\prime} b_{k}^{\prime}=\varnothing$ if $j \neq k$.

If we fix a set $\left\{b_{j}\right\}$ of representatives of $\left\{b_{j}^{\prime}\right\}$ in $G$ and an element $a$, representative of $a^{\prime}$ in $G$, then $\left\{a^{i} b_{j}\right\} \quad(0 \leqq i \leqq n-1)$ is a complete set of representatives of $G^{\prime}$ in $G$, hence $\left\{b_{j}^{-1} a^{-i}\right\}$ is also. Thus both sets $\left\{a^{i} b_{j}\right\}$ and $\left\{b_{j}^{-1} a^{-i}\right\}$ form bases of $K(G)$ over $K(C)$, and

$$
\left\{a^{i} b_{k} \otimes\left(b_{s}^{-1} a^{j}\right)^{*}\right\}
$$

is a basis of $G^{e}$ over $K(C)$.

Let $\alpha=\left(1 \otimes a^{*}-a \otimes 1^{*}\right)$ and suppose that, for some $\gamma \in G^{e}$, we have $\alpha \gamma=0$. Let us call $M_{k l}$ the $K(C)$-submodule of $G^{e}$ spanned by the set $\left\{a^{i} b_{k} \otimes\left(b_{l}^{-1} a^{-j}\right)^{*}\right\} \quad(0 \leqq i, j \leqq n-1, k, l$ fixed $)$, then $G^{e}$ is the direct sum of the set $\left\{M_{k l}\right\}$. It is easily seen that, if $\delta \in M_{k l}$, then $\alpha \delta \in M_{k l}$, hence, if we write $\gamma=\sum \beta_{k l}\left(\beta_{k l} \in M_{k l}\right)$, from $\alpha \gamma=0$ we obtain $\alpha \beta_{k l}=0$. Let

$$
\beta_{s t}=\sum k_{i j s t} a^{i} b_{s} \otimes\left(b_{t}^{-1} a^{-j}\right)^{*},
$$

then $\alpha \beta_{s t}=0$ implies $k_{i i s t}=k_{00 s t}(0 \leqq i \leqq n-1)$.

Now, since $K(G)$ is $G^{e}$-flat, [3, VI, Example 6] assures the existence of elements $\lambda_{u} \in G^{e}, r_{u} \in K(G)$ such that $\alpha \lambda_{u}=0$ and $\sum \lambda_{u} r_{u}=1$. If we call $\gamma=\sum \lambda_{u}\left(1 \otimes r_{u}^{*}\right)$, we find $\alpha \gamma=0$ and $\gamma \cdot 1=1$.

Then, $\gamma=\sum \beta_{s t}, \alpha \beta_{s t}=0$, with $\beta_{s t}$ having the form (2), thus

$$
\sum_{s t} \sum_{i j} k_{i j s t} a^{i} b_{s} b_{t}^{-1} a^{-j}=1 \quad(0 \leqq i, j \leqq n-1)
$$

with $k_{i i s t}=k_{00 s t}$. Since $K(G)=\sum \oplus K(C) a^{i} b_{s}$, the sum (3) need only be extended over those terms for which

$$
a^{i} b_{s} b_{t}^{-1} a^{-j} \in C \text {. }
$$

This last condition shows that $b_{s}^{\prime} \equiv b_{t}^{\prime} \bmod Z^{\prime}$, hence $b_{s}=b_{t}$ and $a^{i-j} \in C$. Since $0 \leqq i, j \leqq n-1$, then $a^{i-j} \in C$ and the fact that $a^{\prime}$ has order $n$ in $G^{\prime}$, imply $i=j$. Thus, the sum (3) is extended only over those terms for which $i=j$ and $b_{s}=b_{t}$, that is,

$$
n \sum_{s} k_{00 s s}=n f=1
$$


and 1 has an inverse $f$ in $K(C)$.

If we write $f=\sum f_{h} g_{h}\left(f_{h} \in K, g_{h} \in C\right.$ with $\left.g_{0}=1\right)$, then $n f=1$ implies $n f_{0}=1$, that is, $n$ has an inverse in $K$.

The following auxiliary results will be used to apply the previous lemmas.

Lemma 5. If $G$ is an abelian group, then either $G$ is a torsion group or it has a free subgroup $M$ such that $G / M$ is a torsion group.

Proof. If $G$ is not a torsion group, then it has elements of infinite order. Let $W$ be the set of subsets $c_{\alpha} \subseteq G$ verifying the following conditions: (1) If $x \in c_{\alpha}$, then the order of $x$ is infinity, (2) the subgroup generated by $c_{\alpha}$ is the direct sum of the (infinite) cyclic groups generated by all the elements $x \in c_{\alpha}$ (i.e., the $x$ 's are independent over the integers).

By ordering $W$ by inclusion, it is trivially true that the join of every ascending chain of $c_{\alpha} \in W$ is an element $c_{\beta} \in W$, then, applying Zorn's lemma, we find a maximal set $c_{\gamma} \in W$. Let us call $M$ the group generated by $c_{\gamma}$.

We must prove, now, that $G / M$ is a torsion group.

If $G=M$, the result is trivial.

If $G \neq M$, suppose $G / M$ is not torsion, and $b^{\prime} \in G / M$ is an element of infinite order.

Let $b$ be a representative of $b^{\prime}$ in $G$. Then $b$ has infinite order. Let $S$ be the subgroup generated by $b$ and $M$.

Every element of $S$ has the form $s=m b^{r}$. If $s=m_{1} b^{r}=m_{2} b^{s}$ then $m_{1} m_{2}^{-1} b^{r-s}=1$. By the homomorphism $G \rightarrow G / M$ we have $b^{r-s}=1$, hence $r=s$, then $b^{r-s}=1$ and $m_{1} m_{2}^{-1}=1$ implies $m_{1}=m_{2}$. This reasoning proves that the representation $s=m b^{r}$ is unique, that is, $S$ $=M \oplus(b)$, hence $c_{\gamma} \cup\{b\} \in W$, contradicting the maximality of $c_{\gamma}$.

THEOREM 1. ${ }^{3}$ If $G$ is a free abelian group and $K$ is a commutative ring without nonzero nilpotent elements, then the group ring $K(G)$ is semisimple.

Proof. Suppose $G$ is an infinite cyclic group. Then the elements of $K(G)$ have the form $\alpha=\sum_{r}^{s} k_{i} g^{i}\left(k_{i} \in K, g\right.$ is the generator of $\left.G\right)$. The subring $T$ of elements $\sum_{0}^{s} k_{i} g^{i}$ is isomorphic with the polynomial ring $K[X]$.

Let $J$ be the radical of $K(G), \alpha=\sum_{r}^{s} k_{i} g^{i} \in J$ and suppose $\alpha \neq 0$. Then, if we call $\beta=\alpha g^{1-r}, \beta=\sum_{1}^{t} k_{i}^{\prime} g^{i}\left(t=s+1-r, k_{i}^{\prime}=k_{i+1-r}\right)$ then $\beta \in T \cap J$ and $\beta \neq 0$. Of course, $T \cdot \beta \subseteq T \cap J$ is an ideal in $T$ and

${ }^{3}$ We had originally proved Theorems 1,2 and 3 over fields of characteristic zero. Our present formulation was suggested by Professor Rosenberg. 
$\gamma \in T \cdot \beta$ implies $\gamma=\sum_{1}^{m} k_{i}^{\prime \prime} g^{i}$. Let $\delta$ be the quasi-inverse of $\gamma$ in $K(G)$. If $\delta=\sum_{r}^{s} h_{i} g^{i}(r \leqq 0)$, by comparing the terms of minimal degree of $\gamma, \delta$ and $\gamma \delta$ in

$$
\gamma+\delta-\gamma \delta=0
$$

we see that $h_{r}=0$. Then $\delta \in T$, that is, $T \cdot \beta$ is a quasi-regular ideal in $T$.

Since $T$ is a polynomial ring over a ring without nonzero nilpotent elements, then $T$ is semisimple [1] contradicting our last result. Thus $\alpha \in J$ implies $\alpha=0$, that is, $K(G)$ is semisimple.

Suppose, now, $G$ is a free abelian group with a finite number, say $n$, of generators. If the semisimplicity of $K\left(G^{\prime}\right)$ is proved in the case $G^{\prime}$ is free abelian with $n-1$ generators, then, since $G=G^{\prime} \oplus Z$, where $Z$ is infinite cyclic, we have $K(G)=\left[K\left(G^{\prime}\right)\right](Z)$ and then our previous reasoning proves $K(G)$ is semisimple.

Let, now, $G$ be any free abelian group, $J$ the radical of $K(G)$ and $\alpha \in J$. Let $X=\left\{x_{i}\right\}$ be the set of generators of $G$; since only a finite number of $x_{i}$ may appear in $\alpha$ with nonzero coefficients, then $\alpha$ is contained in $K\left(G^{\prime}\right)$ where $G^{\prime}$ is a free abelian group generated by a finite subset $Y$ of $X$. Then $K\left(G^{\prime}\right) \cdot \alpha \subseteq J \cap K\left(G^{\prime}\right)$ is an ideal in $K\left(G^{\prime}\right)$ containing $\alpha$, and $K(G)$ is the group algebra $\left[K\left(G^{\prime}\right)\right]\left(G^{\prime \prime}\right)$ where $G^{\prime \prime}$ is the free abelian subgroup of $G$ generated by the elements of $X-Y$.

If $\gamma \in K\left(G^{\prime}\right) \cdot \alpha$ and $\delta$ is the quasi-inverse of $\gamma, \delta=\sum f_{i} \theta_{i}\left(f_{i} \in K\left(G^{\prime}\right)\right.$, $\left.\theta_{i} \in G^{\prime \prime}\right)$ then, if we call $\theta_{1}=1$, from $\gamma+\sum f_{i} \theta_{i}-\sum \gamma f_{i} \theta_{i}=0$ we obtain $\gamma+f_{1}-\gamma f_{1}=0$, that is, $\gamma$ has a quasi-inverse in $K\left(G^{\prime}\right)$, then $K\left(G^{\prime}\right) \cdot \alpha$ is a quasi-regular ideal in $K\left(G^{\prime}\right)$. Since $G^{\prime}$ is finitely generated, the semisimplicity of $K\left(G^{\prime}\right)$ previously proved implies $\alpha=0$, then $J=0$ and $K(G)$ is semisimple.

THEOREM 2. If $G$ is any commutative group and $K$ is a semisimple commutative algebra over the rational numbers, then $K(G)$ is semisimple.

Proof. If $G$ is a torsion group, we apply Lemmas 1 and 3 with $C=(1)$, then $K(C) \cong K$ is semisimple by hypothesis. If $G$ is not torsion, then we take $C=M$ ( $M$ as obtained in Lemma 5), then Lemma 5 and Theorem 1 assert $K(C)$ is semisimple, the semisimplicity of $K(G)$ following as in the previous case.

Theorem 3. Let $G$ be a group and $Z$ its center. If $G / Z$ is locally finite and $K$ is a semisimple commutative algebra over the rationals, then $K(G)$ is semisimple.

Proof. The semisimplicity of $K(Z)$ is assured by Theorem 2, then Lemmas 3 and 1 give the result of the theorem. 
REMARKs 1 . In Theorems 2 and 3, if $G$ is a torsion group, we may weaken the condition of $K$ to be an algebra over the rationals by imposing $K$ to be uniquely divisible by the orders of each element in $G$.

2. In the same theorems, if $G$ is not torsion (in Theorem 3, the local finiteness of $G / Z$ implies $Z$ is not torsion) then, in order to apply Theorem 1 , it is sufficient $K$ to have no nonzero nilpotent elements.

3. From [3, X Theorem 6.2], we have that $K$-w. $\operatorname{dim} K(G)=0$ if and only if w. $\operatorname{dim}_{K(G)} K=0$. Thus, [2, Proposition 6] follows from Lemmas 3 and 4 . Since w. gl. $\operatorname{dim} K(G)=0$ implies w. $\operatorname{dim}_{K(G)} K=0$, hence $K$-w. $\operatorname{dim} K(G)=0$, then Lemmas 3 and 4 are applicable and we find a new proof of Theorem 9 of [2].

\section{REFERENCES}

1. S. A. Amitsur, Radicals of polynomial rings, Canadian J. Math. vol. 8 (1956) pp. $355-361$.

2. M. Auslander, On regular group rings, Proc. Amer. Math. Soc. vol. 8 (1957) pp. 658-664.

3. H. Cartan and S. Eilenberg, Homological algebra, Princeton University Press, 1956.

4. M. Harada, Note on the dimension of modules and algebras, J. Inst. Polytechnics, Osaka City Univ. vol. 7 (1956) pp. 17-27.

5. N. Jacobson, The radical and semisimplicity for arbitrary rings, Amer. J. Math. vol. 67 (1945) pp. 300-320.

6. A. Rosenberg and D. Zelinsky, Cohomology of infinite algebras, Trans. Amer. Math. Soc. vol. 82 (1956) pp. 85-98.

7. J. von Neumann, On regular rings, Proc. Nat. Acad. U.S.A. vol. 22 (1936) pp. 707-713.

Universidad Nacional de la Plata and

The Institute for Advanced Study 